\title{
Correlation of CT and MR with Impedance Monitoring and Histopathology in Stereotactic Biopsies
}

\author{
J. Gorecki , E.J. Dolan, R.R. Tasker and W. Kucharczyk
}

\begin{abstract}
Magnetic Resonance Imaging (MR) and Computer Assisted Tomography (CT) guided stereotaxis combined with intraoperative impedance monitoring and multiple sequential biopsies provides the opportunity to correlate the results of radiologic imaging with impedance and histopathology. The authors present the methods used and preliminary results obtained from 30 stereotactic biopsies with complete correlation in 12 cases. Impedance changes correlate accurately with lesion margins as defined by histology. CT images of enhancing lesions correlate quite closely to the histopathologic lesion margins whereas the appearance of the lesion on MR images is often larger than subsequently identified by either impedance or at pathologic examination. Impedance monitoring is a useful adjunct to stereotactic biopsy as it helps to accurately define lesion margins and can help direct the choice of biopsy sites.

RÉSUMÉ: Corrélation des examens par CT et MR avec le monitoring de l'impédance et l'histopathologie dans les biopsies stéréotaxiques La stéréotaxie guidée au moyen de l'imagerie par résonance magnétique (MR) et de la tomographie assistée par ordinateur (CT) combinée au monitoring de l'impédance peropératoire et à des biopsies séquentielles multiples fournissent l'occasion de corréler les résultats de l'imagerie radiologique avec ceux de l'impédance et de l'histopathologie. Les auteurs présentent les méthodes utilisées et les résultats préliminaires obtenus à partir de 30 biopsies prélevées sous stéréotaxie avec corrélation complète dans 12 cas. Les changements d'impédance ont une corrélation précise avec les limites de la lésion telles que définies par l'examen histologique. Il y a une corrélation assez étroite entre les images par CT de lésions qui rehaussent au produit de contraste et les limites histopathologiques de ces lésions, alors que les images par MR montrent souvent des lésions plus étendues que ce qui est constaté ultérieurement lors de l'examen par impédance ou à la pathologie. Le monitoring par impédance est un complément utile à la biopsie stéréotaxique parce qu'il aide à définir précisément les limites des lésions et peut aider à choisir les sites de biopsie.
\end{abstract}

Can. J. Neurol. Sci. 1990; 17:184-189

The technique of stereotactic neurosurgery was introduced in humans in 1946 by Spiegel et al. ${ }^{1}$ Differences in the conductivity between normal and pathological tissue were well documented as early as $1921.2,3$ Attempts to localize brain tumours by the measurement of tissue impedance (electrical resistance) began in $1921^{2}$ and in $1923 \mathrm{Grant}^{4}$ reviewed the impedance patterns in 12 operative cases, fresh autopsy brains, formalin preserved brains, and 60 trials on animals, concluding that the measurement of electrical resistance (impedance) was helpful in locating tumours not reaching the cortical surface. This was subsequently corroborated by others. ${ }^{5,6,7.8}$ Most of this work was done before computerized tomography (CT) and magnetic resonance (MR) imaging was available. For this reason it was not possible to clearly distinguish if the impedance changes occurred at the tumour edge, or only after the electrode had entered the tumour.

The introduction of CT and MR scanning has dramatically changed the way stereotactic procedures are carried out.9,10
Localizing tumours is now much easier and safer. This has resulted in a recent surge in the use of stereotactic biopsy for deeply located lesions. ${ }^{11}$ At the same time interest in impedance monitoring has fallen, localization no longer being the problem it was in the pre-CT era. However, debate remains as to the exact nature of the lesions being demonstrated by CT or MR images, particularly in view of the fact that respective CT and MR images of the same lesion can look so different. DaumasDuport et al classified glial tumours based on their pattern of cellular invasion as determined by serial stereotactic biopsies. ${ }^{12}$ Type I tumours had a nodule of tumour with no distant isolated cells, and type II tumours also had a tumour nodule, but with malignant cells spreading distally from the tumour edge. Type III tumours had no nodule and consisted of isolated malignant cells scattered throughout the otherwise normal brain parenchyma. Earnest et al ${ }^{13}$ have suggested that enhanced CT scans identify the solid component of the tumour while the MR is bet-

From the Division of Neurosurgery and Department of Radiology, Toronto General Hospital and University of Toronto, Toronto

Reprint requests to: E.J. Dolan, Division of Neurosurgery, Toronto General Hospital, Room 222, 14 Eaton North, 200 Elizabeth Street, Toronto, Ontario, Canada M5G 2C4 
ter at delineating the extent of the spread of malignant cells However, they also suggested that isolated malignant cells can be identified by histopathological examination outside the boundaries defined for the tumours by either CT or MR. Stereotactic impedance monitoring in conjunction with radiologic investigations could aid in more accurate determination of the edge of the tumour and give a better idea of the cellular pattern of the tumour.

For this reason, we have carried out sequential stereotactic biopsies along specifically calculated trajectories as defined by both CT and MR imaging and correlated these with intraoperative impedance monitoring along the biopsy trajectory. Post operative MR scanning was used to identify the exact biopsy trajectory and location of the biopsy. The data thus collected allowed correlation between the histopathology, diagnostic imaging, and the electrical conductivity.

\section{METHODS}

Thirty consecutive patients were investigated as follows. All patients were studied preoperatively with either enhanced CT or MR. Steroids (dexamethasone) were used perioperatively. On the morning of the biopsy the Leksell stereotactic frame was applied with the patient seated in a wheel chair, with the aid of local anaesthesia. In the radiology department axial CT (GE 9800) and MR (GE Signa 1.5 Tesla scanner) images were then obtained for targeting. The frame adaptors hold the Leksell frame in a fixed position rectilinear to the CT and MR imaging gantries making it possible to obtain images in the same axial plane from both techniques. This allowed direct comparison of the CT and MR images. The MR further allowed sagittal images of the lesion. A trajectory was chosen on these sagittal images and angled coronal or oblique images were then produced through the proposed biopsy trajectory (Figure 1). This allowed accurate determination of the structures that would be traversed by the exploring electrode.

The patient was then readied for the actual biopsy, carried out in the operating room under local anaesthesia. Biopsies were performed through a twist drill craniotomy. The dura was opened with a sharp needle. Following this the first pass along the trajectory to the target was carried out with a bipolar coaxial electrode to record tissue impedance. This is the same electrode used to produce thalamic radiofrequency lesions, and has a $3 \mathrm{~mm}$ exposed tip and an outer diameter of $1 \mathrm{~mm}$. A flat metal plate fastened to the patient's ipsilateral arm acted as the electrical ground. Impedance was measured at $50 \mathrm{kHz}$ (OWL Universal RF System, Diros Technology Inc., Toronto) starting at the pial surface, and measured every $5 \mathrm{~mm}$ along the trajectory until $1 \mathrm{~cm}$ to $1.5 \mathrm{~cm}$ beyond the target. ${ }^{7}$ Readings were taken by advancing the needle in $5 \mathrm{~mm}$ increments and recording the impedance after it had become stable. Attempts at repeated impedance measurements along the same trajectory produced different and unreliable readings as has been noted previously. 5,6

Serial biopsies were then taken with a Sedan biopsy needle (provided by Dr. P.J. Kelly, Mayo Clinic, Rochester) in $5 \mathrm{~mm}$ advancements starting proximal to the lesion margin as defined by imaging and impedance. This produced a core of tissue $10 \mathrm{~mm}$ long and $1.0 \mathrm{~mm}$ in diameter. ${ }^{15}$ The tissue was labelled, the proximal end identified, and permanent paraffin longitudinal sections obtained.

Post-operative MR scanning was then performed in oblique planes to produce images in the plane of the biopsy trajectory such that the trajectory was clearly visible (Figure 2). It was a relatively simple matter to correlate imaging findings with the impedance and biopsy results from these scans. It was more difficult to obtain trajectory data from CT scans, particularly if the biopsy trajectory was an oblique one. The trajectory could be plotted on the scout images by hand, and then localized on the various axial images by hand or using the correlate software package on the CT scanner. Comparison was then possible between CT, MR impedance and the histopathology.

\section{Results}

Stereotactic biopsies were performed on 30 patients (Table 1) using the above techniques over the preceding 18 months. Due to early ongoing changes in the specific procedures impedance data was available for only 21 cases and complete impedance,
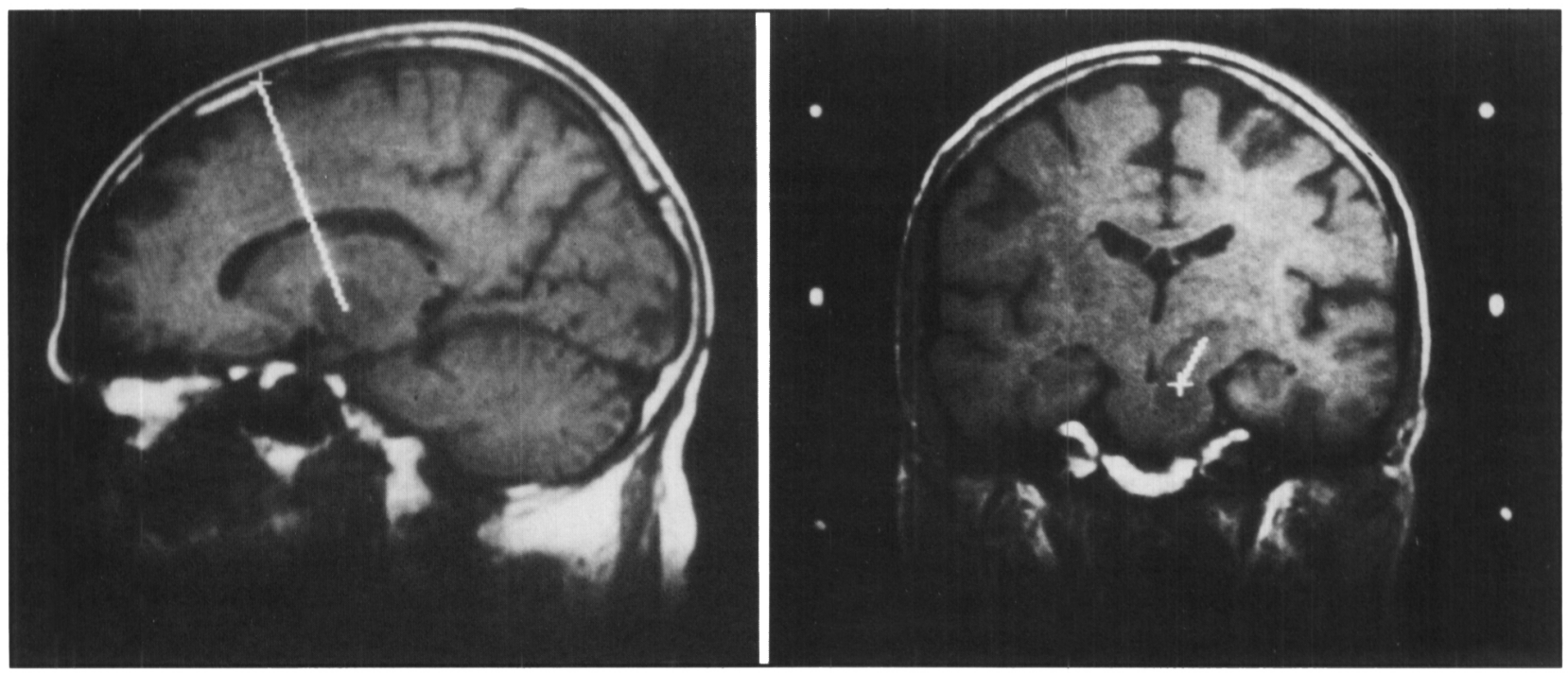

Figure I - Pre-operative MR allows preplanning of biopsy trajectories in two orthogonal planes. 

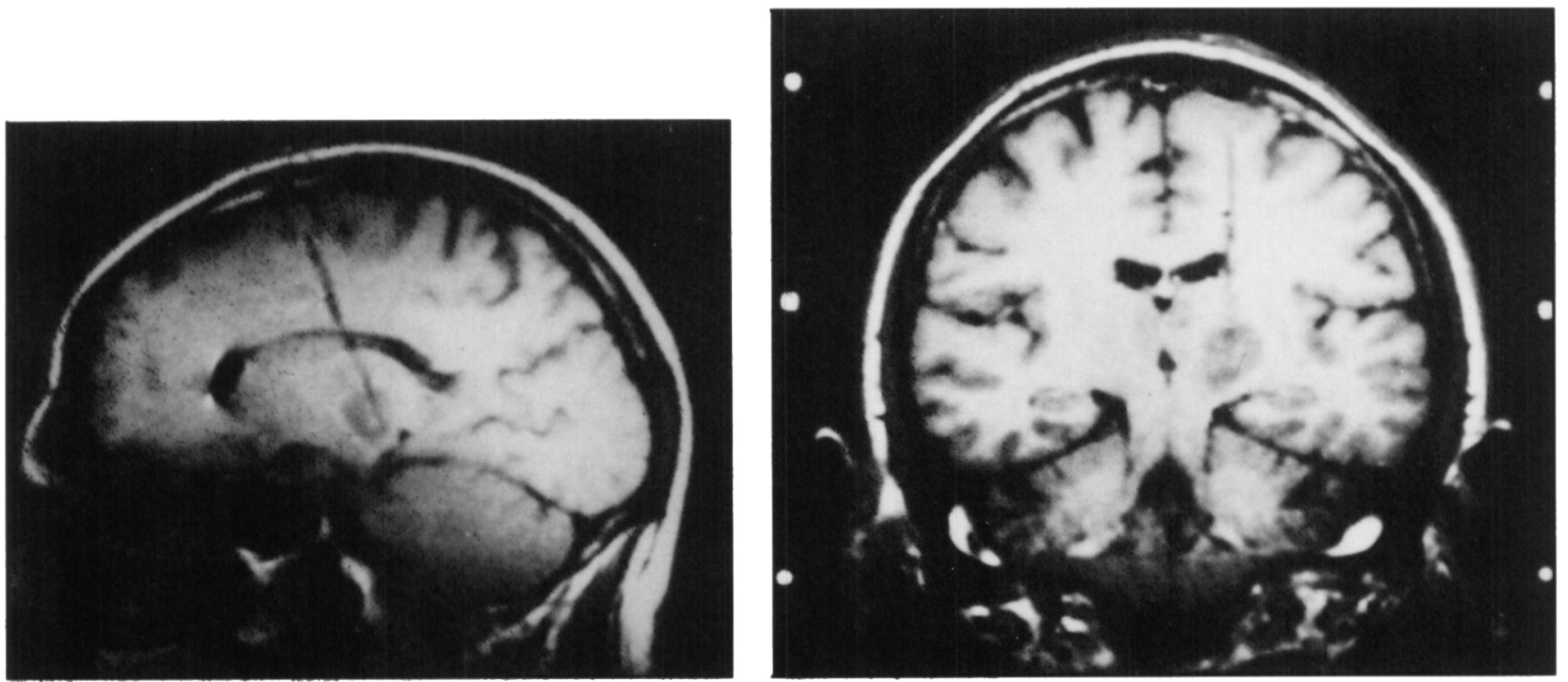

Figure 2 - The post-operative MR study clearly shows the biopsy tract.

\begin{tabular}{lccc}
\hline Table 1: Summary of Histopathology & \\
\hline \hline Pathology & $\begin{array}{c}\text { Number of } \\
\text { Cases }\end{array}$ & $\begin{array}{c}\text { Impedance Data } \\
\text { Available }\end{array}$ & $\begin{array}{c}\text { Impedance CT } \\
\text { and MR Data } \\
\text { Available }\end{array}$ \\
\hline Glial Tumour & 15 & 10 & 9 \\
Craniopharyngioma & 2 & 2 & 0 \\
Lymphoma & 3 & 1 & 1 \\
Gliosis & 2 & 2 & 1 \\
Toxoplasmosis & 2 & 2 & 0 \\
Cavernous hemangioma & 2 & 2 & 0 \\
Cysticercosis & 1 & 1 & 0 \\
Arachnoid Cyst & 1 & 0 & 0 \\
Herpes Encephalitis & 1 & 1 & 1 \\
Aids Encephalitis & 1 & 0 & 0 \\
\hline & 30 & 21 & 12 \\
\hline
\end{tabular}

always fell when passing into cerebrospinal fluid (CSF) which had an mean impedance of 300-400 ohms. The impedance in thalamus and basal ganglia was intermediate between grey and white matter with a mean impedance of 450-550 ohms.

Impedance changes could be assessed at the lesion edge in 21 cases. The most common pattern - a dramatic fall in impedance upon entry into the lesion 7 - was seen in 15 cases, and was true in all but 1 of the tumour cases. In one tumour case the impedance rose. This recording was of poor quality and technical factors may have played a role. In 5 cases there was no identifiable change in impedance. This was expected in two cases ( 1 glioblastoma, and 1 gliosis) as the lesions were so extensive as to occupy the entire biopsy trajectory. A cysticercosis cyst was aspirated through the biopsy probe in spite of a steady impedance in another case. Interestingly, two patients who harboured cavernous hemangiomas also showed no changes in impedance. Others have commented on the lack of change of impedance on entering intracerebral hematomas. ${ }^{16}$

The 30 patients demonstrated a typical mix of CT appearMR and CT and histopathology data was obtained for preliminary analysis for 12 cases. Most lesions were deep. The spectrum of pathology is presented in Table 1 . The great majority of patients had glial tumours.

In order to confirm the accuracy of the MR scanning, target coordinates for the lesion, as well as easily identified anatomical landmarks were chosen on both the MR and CT images and compared. Targets fell within $\pm 1 \mathrm{~mm}$ and there was excellent reproducibility with a coefficient of correlation of .99 (Figure 3 ).

It was quite clear that the pattern of impedance was far more valuable than the absolute values obtained. The direction of the change in impedance, in passing from one tissue to the next, was consistent and reproducible between patients. Therefore, with an understanding of the 3-dimensional anatomy of the proposed trajectory it was easy to be confident of where the exploring electrode was at any given point on the trajectory. There was however, considerable variation in absolute impedance values, making it difficult to interpret mean values calculated for the group of patients studied. Impedance generally rose in passing from grey into white matter, and although the mean impedance in white matter was $500-600 \mathrm{ohms}$ and in grey matter was 600 700 ohms, there was considerable overlap. The impedance ances, with 5 ring enhancing lesions, 20 diffusely enhancing lesions, 4 hypodense lesions, and 1 cystic lesion (craniopharyngioma). Of the 12 fully analyzed patients there were 3 ring enhancing lesions, 5 diffusely enhancing, and 3 hypodense lesions, and 1 hypodense craniopharyngioma cyst. In 9 of the 12 cases the edge of the lesion as defined by CT corresponded to the limits as defined both by the impedance, and by histopathologic examination. In 5 cases of glioblastoma multiforme (3 ring enhancing lesions, 1 diffusely enhancing lesion and 1 hypodense lesion) and a case each of cerebral lymphoma, herpes encephalitis and a craniopharyngicma cyst, a sharply demarcated edge was seen on the CT. The glial tumours would correspond to the Daumas-Duport type I lesion, 12 in that the tumour edge is sharply seen on the CT, and clearly identified on the impedance recordings, and histopathology. In 2 astrocytic tumours ( 1 astrocytoma, and 1 glioblastoma multiforme) the diffuse enhancement of the lesion seen on CT was larger than defined by the impedance measurements. Pathological examination showed solid tumour beginning at the zone where impedance fell, but some scattered tumour cells could be seen 


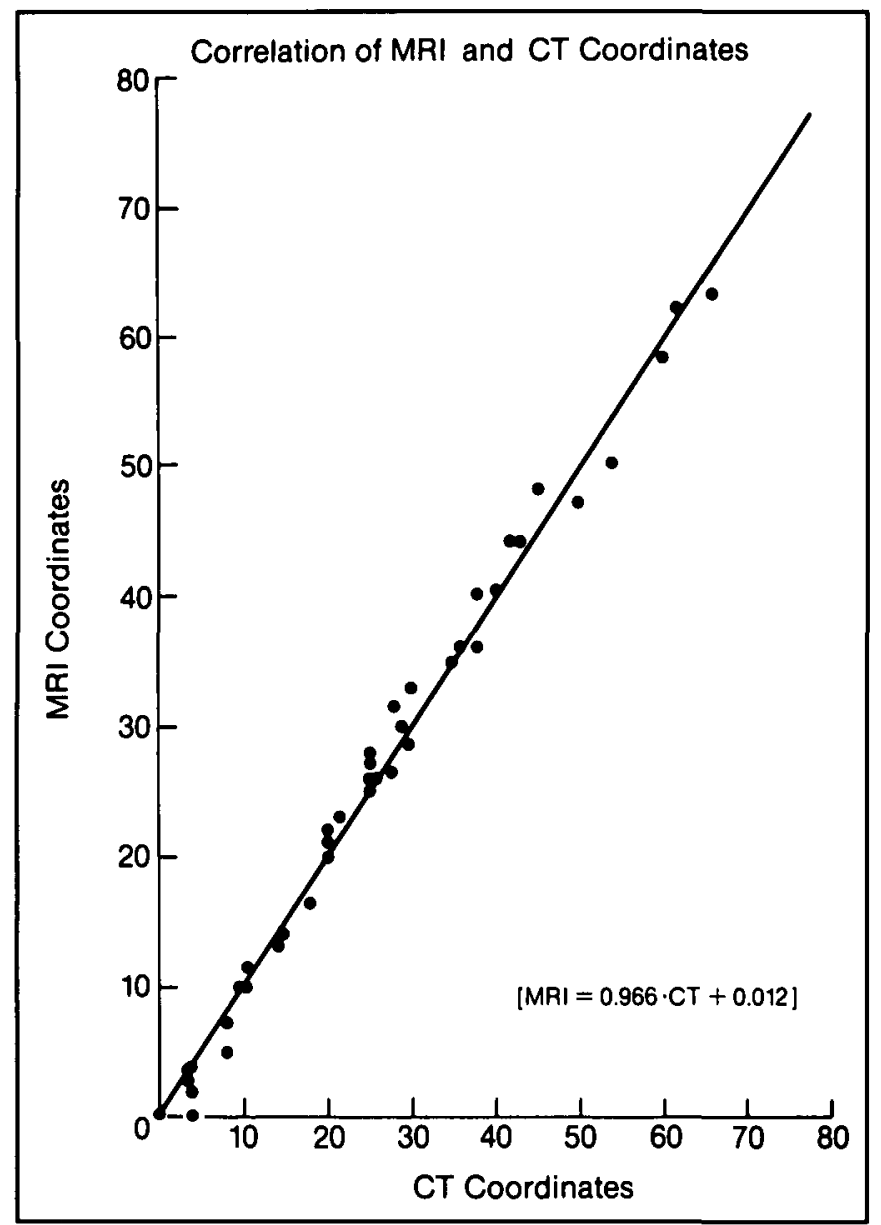

Figure 3 - Correlation between $C T$ and $M R$ coordinates for target points, and brain structures (e.g. fornix, lateral ventricle) at various distances from the centre of the Leksell frame. $r=0.99$.

distal to this, representing a Daumas-Duport type II lesion. The impedance clearly identified the solid tumour nodule, while the CT images demarcated the extent of spread of distal tumour cells. In another case, while the impedance change corresponded exactly with the enhancing CT margin of the lesion, this margin as defined by histology represented a transition from normal brain tissue to gliotic tissue surrounding a tumour. In a case with a diffuse lesion seen on CT scan no changes of impedance were seen along the biopsy trajectory, and all biopsy specimens were abnormal, revealing extensive gliosis and changes compatible with methotrexate cerebral toxicity.

The MR images showed a signal decrease on the $\mathrm{Tl}$ images in all cases, and a signal increase on the T2 weighted images. The T2 weighted images tended to show the lesion as larger than either the $\mathrm{Tl}$ weighted images or the CT scan images. In 5 of our cases (4 cases of glioblastoma multiforme, and a case of lymphoma) the MR T1 image edges corresponded to the histological edge, and also to the tumour edge as defined by impedance. In the one case of a gliotic edge surrounding tumour, this edge was identical on the MR T1 and T2 images, and corresponded to the edges as defined by CT and impedance measurement as mentioned above. Five cases revealed MR T1 images which showed lesion volumes larger than recognized by either CT or histopathology. The impedance edge in these cases corre- sponded more closely to the histopathology edge. Presumably the MR images reflect the changes in water content of the brain tissue surrounding the lesion. ${ }^{15}$ In the case of the methotrexate effect (gliosis) the lesion was so extensive that all biopsy specimens were abnormal, the CT and MR scans showed a diffuse abnormality, and the impedance measurements showed no changes along the biopsy trajectory.

A typical example of a case of a thalamic astrocytoma is shown in Figure 4. This case revealed a hypodense lesion on $\mathrm{T} 1$ MR images. It was possible to clearly follow the rise in impedance on moving from cortex to white matter, and then the precipitous fall in impedance upon passing into the CSF of the ventricles. The impedance rose again on entering the thalamus, and fell markedly on reaching the lesion. A second example from a patient with a glioblastoma multiforme (Figure 5) presented a ring enhancing lesion on CT. The impedance was seen to fall at the edge of this enhancing ring. Histology confirmed tumour at the site of the enhancing ring, with a sharp demarcation from normal tissue, and a necrotic centre.

In two of our cases of ring enhancing lesions, impedance initially fell at the edge of a lesion, then rose on further advancement into necrotic area as defined by histopathology. This is the opposite to the results seen by Broggi and Franzani, in a similar ring enhancing lesion. 17

\section{Discussion}

Impedance measurements recorded during stereotactic biopsies follow characteristic patterns. There is a rise in impedance in passing from grey into white matter, and mixed grey and white matter tissues have an intermediate impedance between that of grey and white matter. Invariably a fall in impedance occurs when CSF is encountered.5,16.18 A fall in impedance is commonly seen when the electrode enters abnormal tissue, and this appears to correspond to the actual lesion edge as defined by histopathology. This pattern can be used to help identify the margins of a variety of lesions. ${ }^{19.20}$ Bullard and Makachinas $^{5}$ with the aid of CT guided stereotaxis showed a variable response at the lesion edge, with 11 cases showing a rise in impedance, and in 2 a fall at the enhancing edge. They had 4 cases with biopsies at the area of increased impedance and found viable tumour, while at the zone with decreased impedance only necrosis was seen. Our results confirm the previously described fall in impedance seen in abnormal tissue, 5.19 but more clearly define that this occurs at the lesion edge, and not in the core of the lesion. While some discrepancy regarding the exact depth of the lesion edge may result from tissue being deformed by the advancing electrode this deformation should amount to less than $2 \mathrm{~mm}$ in most tissues, ${ }^{14}$ and considerably less if the tissue is soft. We have only noticed this to be a problem in one case of a craniopharyngicma cyst. A feeling of pain inside the patient's head was produced on reaching the cyst wall edge as calculated from the CT and MR scans, but the cyst proper was not entered for an additional $7 \mathrm{~mm}$ at which point the pain was relieved. We believe the pain was from traction on surrounding structures as the cyst wall was pushed away from the electrode.

There was generally good correlation between the lesion edge as seen by CT imaging, pathology, and impedance, however, in two cases the CT showed a larger area affected. MR 


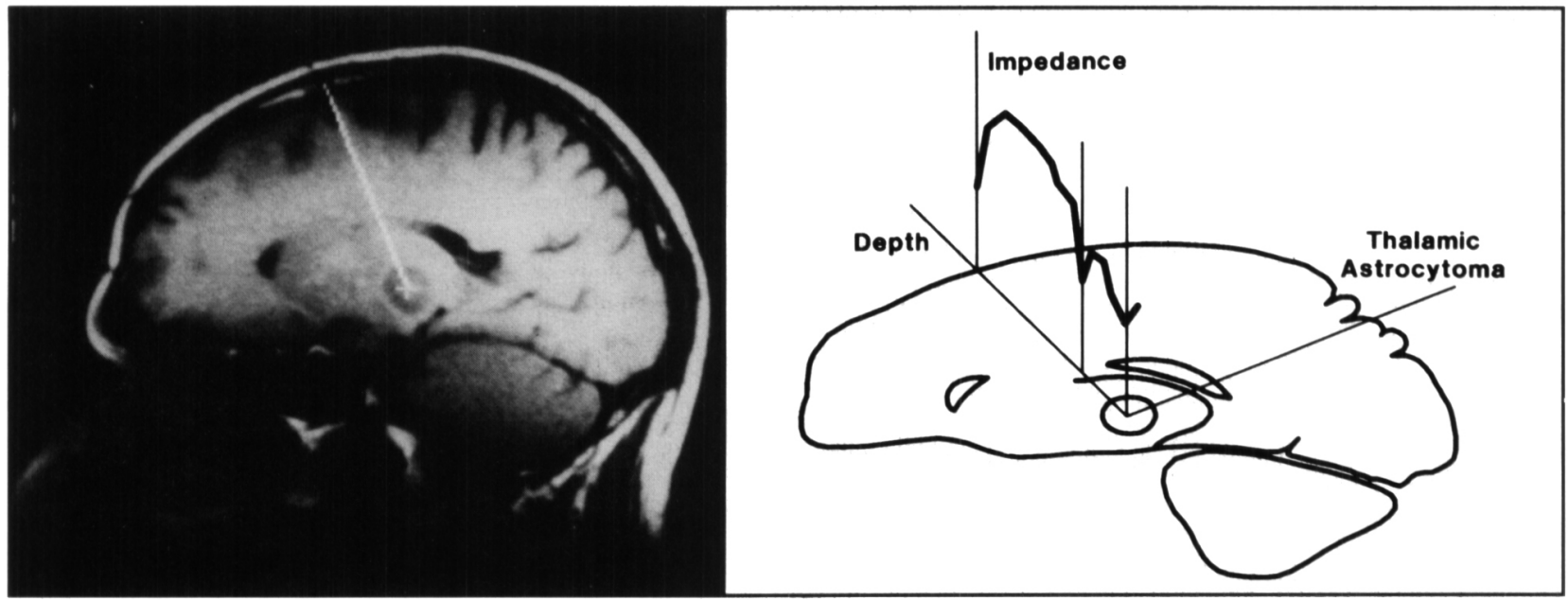

Figure 4-An example of an impedance profile (right) for a thalamic astrocytoma. A rise in impedance occurs in moving from cortical grey matter into white matter, and a fall on entering CSF. The impedance in thalamic tissue is intermediate between that of grey and white matter. There is a fall in impedance at the tumour edge.

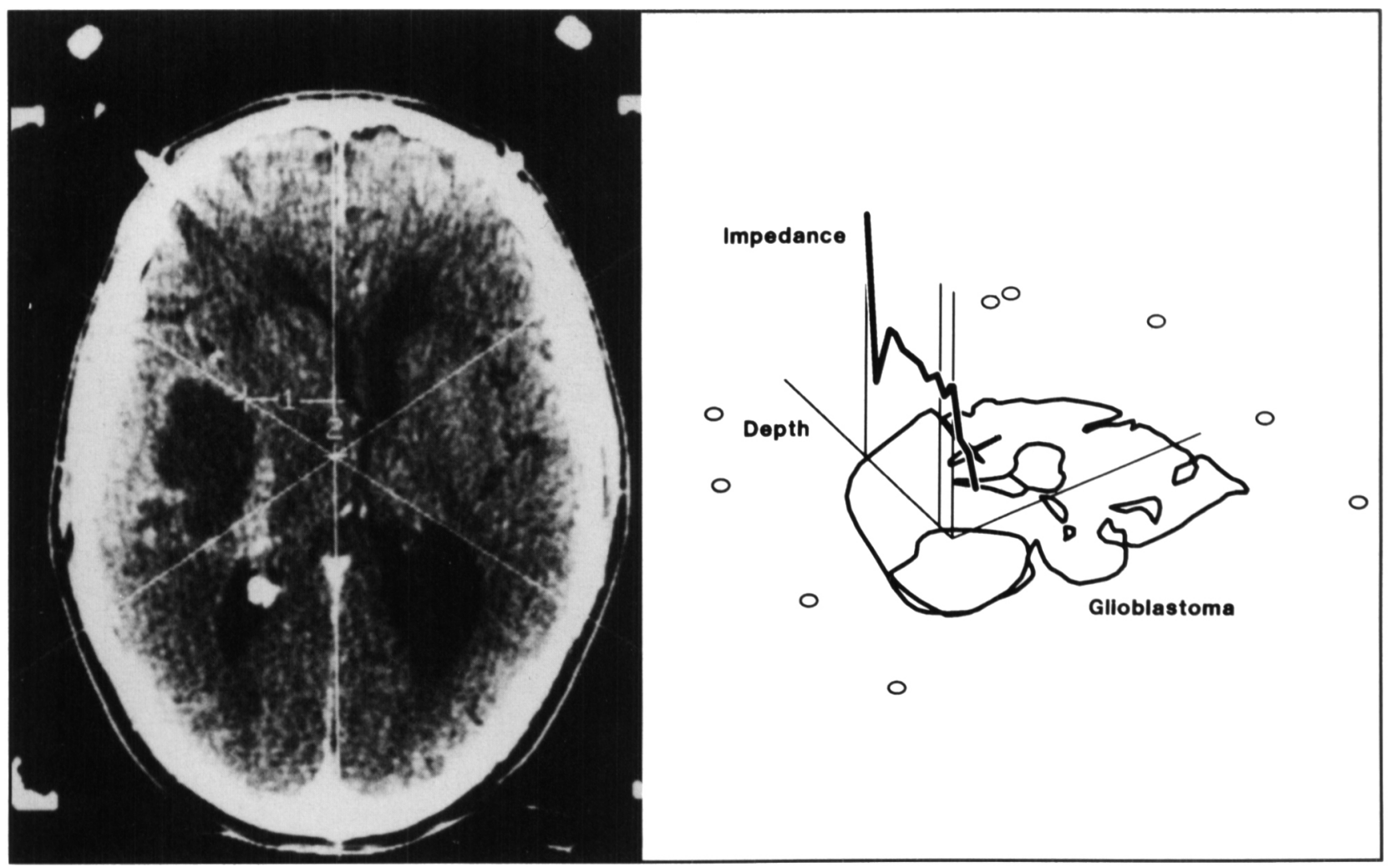

Figure 5-The impedance profile (right) of a glioblastoma multiforme presenting as a ring enhancing lesion on CT (left), showing the fall of impedance at the enhancing edge of the lesion. The small circles on the diagram represent the Leksell frame and fiducial markers. 
imaging, particularly the T2 images tended to show abnormalities over a much larger area than confirmed by pathology. In one case the lesion margins as defined by impedance, CT, and MR imaging corresponded to an area of surrounding gliosis, and not the tumour proper. Therefore, at this stage, histology as determined at biopsy remains the only way to confirm the true limits of the lesion.

In our hands serial stereotactic biopsy under local anaesthesia using the Sedan biopsy needle has been a safe, reliable technique for obtaining tissue. There were four complications in the 30 cases, 3 transient worsenings of preexisting hemiparesis and one small haematoma at the biopsy site. All resolved with no further treatment. Serial biopsies allow for better sampling, especially in astrocytic tumours and for better definition and classification of tumour margins. ${ }^{21}$ Impedance monitoring is a valuable aid in the choice of biopsy sites, ${ }^{17}$ and in particular in identifying lesion margins.

MR enhances the safety of the stereotactic biopsy. It allows a clearer understanding of the proposed (and actual) biopsy trajectory in 3-dimensional space. This makes planning of the biopsy trajectory much more flexible, providing the ability to see a variety of planned trajectories, and enabling the choice of a trajectory that avoids vascular structures, and eloquent areas, and specifically passes through other areas. The limiting factor is the length of time required for acquisition of the MR and CT images.

The use of computer graphics to trace out the lesions as seen on diagnostic imaging, and to plot the biopsy trajectory as well as the impedance record make it technically much easier and more accurate to carry out the desired comparisons.

\section{ConClusions}

MR is a valuable adjunct to stereotaxy, allowing accurate probe trajectory planning which we believe improves the safety of stereotactic biopsy.

Impedance monitoring is an invaluable intraoperative tool for stereotactic biopsy procedures. The impedance pattern has proved more valuable than absolute values. Impedance is higher in white matter than grey matter, with intermediate values in basal ganglia and thalamus and low values in CSF. Impedance tends to fall at the lesion margins, allowing a more accurate discrimination of these margins and improved ability to direct the choice of biopsy sites.

The present study suggests that correlating the impedance measurements with the CT and MR images can predict the type of tumour edge (focal or diffuse). Further studies will be necessary before one can determine if correlation of MR, CT and impedance data can accurately predict the type of tumour edge intraoperatively.

\section{REFERENCES}

1. Spiegel EA, Wycis HT, Marks M, et al. Stereotaxic apparatus for operations on human brain. Science 1947; 106: 349-350.

2. Meyer AW. Methode zum auffinden von hirntumoren bei der trepanation durch elektrische widerstandsmessung. Zlb Chir 1921; 48: 1824-1826.

3. Crile GW, Hosmer HR, Rowland AF. The electrical conductivity of animal tissue under normal and pathological conditions. Am J Physiol 1922; Feb: 59-106.

4. Grant FC. Localization of brain tumours by determination of the electrical resistance of the growth. J Am Med Assoc 1923; 81: 2169-2171.

5. Bullard DE, Makachinas TT. Measurement of tissue impedance in conjunction with computed tomography-guided stereotactic biopsies. J Neurol Neurosurg Psychiatry 1987; 50: 43-51.

6. Organ LW, Tasker RR, Moody NF. The impedance profile of the human brain as a localization technique in stereoencephalotomy. Conf Neurol 1967; 29: 192-196.

7. Organ LW, Tasker RR, Moody NF. Brain tumour localization using an electrical impedance technique. J Neurosurg 1968; 28: 35-44.

8. Robinson BW, Bryan JS, Rosvold HE. Locating brain structures Extensions to the impedance method. Arch Neurol 1965; 13: 477-486.

9. Kelly PJ, Kall B, Goerss S. Stereotactic CT scanning for the biopsy of intracranial lesions and functional neurosurgery. Appl Neurophysiol 1983; 46: 193-199.

10. Leksell L, Leksell D, Schwebel J. Stereotaxis and nuclear magnetic resonance. J Neurol Neurosurg Psychiatry 1985; 48: 14-18.

11. Gildenberg PL. Stereotactic neurosurgery and computerized tomographic scanning. Appl Neurophysiol 1983; 46: 170-179.

12. Daumas-Duport C, Monsaigneon V, Blond S, et al. Serial stereotactic biopsies and CT Scan in gliomas: Correlative study in 100 astrocytomas, oligoastrocytomas and oligodendrocytomas. J Neuro-Oncology 1987; 4: 317-328.

13. Earnest F, Kelly PJ, Scheithauer BW, et al. Cerebral astrocytomas: Histopathologic correlation of MR and CT contrast enhancement with stereotactic biopsy. Radiology 1988; 166: 823-827.

14. Robinson BW. Localization of intracranial electrodes. Exp Neurol 1962; 6: 201-223.

15. LeBas JF, Leviel JL. Decorps M, et al. NMR relaxation times from serial stereotactic biopsies in human brain tumours. $\mathrm{J}$ Ccmput Assist Tomogr 1984; 8: 1048-1057.

16. Becker DP, Robert CM, Seelig J. A simplified method for electrical impedance monitoring in brain tumour localization. J Neurosurg 1970; 32: 375-377.

17. Broggi G, Franzani A. Value of serial stereotactic biopsies and impedance monitoring in the treatment of deep brain tumours. J Neurol Neurosurg Psychiatry 1981; 44: 397-401.

18. Gildenberg PL, Zenes C, Flitter $M$, et al. Impedance measuring device for detection of penetration of the spinal cord in anterior percutaneous cervical cordotomy. J Neurosurg 1969; 30: 87-92.

19. Benabid AL, Persat JC, Chirossel JP, et al. Correlative study between computerized transverse scanning and stereoimpedoencephalography in space-occupying lesions of the brain. Acta Neurochir 1979; 46: 219-232.

20. Laitinen LV, Johansson GG. Locating human cerebral structures by the impedance method. Conf Neurol 1967; 29: 197-201.

21. Waltregny A, Petrov V, Brotchi J. Serial stereotaxic brain biopsies. Acta Neurochir Suppl 1974; 21: 221-226. 